\title{
A non-associative quantum mechanics
}

\section{Vladimir Dzhunushaliev}

\author{
Dept. Phys. and Microel. Engineer., Kyrgyz-Russian Slavic University, Bishkek, Kievskaya Str. 44, \\ 720021, Kyrgyz Republic \\ e-mail: dzhun@hotmail.kg
}

\begin{abstract}
A non-associative quantum mechanics is proposed in which the product of three and more operators can be non-associative one. The multiplication rules of the octonions define the multiplication rules of the corresponding operators with quantum corrections. The self-consistency of the operator algebra is proved for the product of three operators. Some properties of the non-associative quantum mechanics are considered. It is proposed that some generalization of the non-associative algebra of quantum operators can be helpful for understanding of the algebra of field operators with a strong interaction.
\end{abstract}

Key words: octonions, non-associative algebra, non-perturbative quantization

\section{INTRODUCTION}

In any modern quantum theory the algebra of quantized fields is defined by canonical commutations/anticommutations relationships between field operators in two different points. It is well known that strictly speaking such algebra can correctly describe the algebra of free fields only. For weakly interacting fields (quantum electrodynamics, for example) we can calculate the n-points Green's functions using Feynman diagram technique and in principle these functions give us full information about interacting fields. But for the strongly interacting fields the situation is another: without the knowledge of the algebra of quantized fields we can not carried out the operator quantization of such theories. The examples of strongly interacting theories are: $\lambda \phi^{4}$ - theory with $\lambda \gg 1$, quantum chromodynamics in some regimes and gravity at the Planck level. Therefore one can formulate one of the main problems in quantum field theory: the formulation of the algebra of quantized fields whose generating relationships are more complicated the canonical relationships. Of course such problem for quantum field theory is very complicated and in this notice we would like to present a non-associative quantum mechanics where we have two relationships generating the algebra of quantum operators: the first one is similar to the ordinary anticommutation rule for the creation and annihilation fermion operators and the second one is the rule for the movement of brackets in the product of three operators.

\section{NON-ASSOCIATIVITY: PRELIMINARY REMARKS}

In this section we would like to remember for reader the first example of a non-associativity in mathematics: octonions and review some attempts to introduce the non-associativity in physics.

At first we would like to give some preliminary in the non-associativity. A vector space is a finitedimensional module over the field of real numbers. An algebra $A$ is a vector space which is equipped with a 
bilinear map $m: A \times A \rightarrow A$ (multiplication) and a nonzero element $1 \in A$ (unit) such that $1 \cdot a=a \cdot 1=a$. The algebra $A$ is non-associative one if

$$
(a \cdot b) \cdot c \neq a \cdot(b \cdot c)
$$

The algebra $A$ is alternative if

$$
(a \cdot a) \cdot b=a \cdot(a \cdot b), \quad(b \cdot a) \cdot a=b \cdot(a \cdot a), \quad(a \cdot b) \cdot a=a \cdot(b \cdot a) .
$$

Later we will omit the multiplication sign (.).

\subsection{The Octonions}

Good and simple introduction for physicists to the octonions is Ref. 1. The octonions are an 8D algebra with the basis $1, \tilde{q}_{i}, \tilde{Q}_{i}, I$ and their multiplication is given in the Table 1 It is easy to see that this algebra

\begin{tabular}{|c|c|c|c|c|c|c|c|}
\hline & $\tilde{q}_{1}$ & $\tilde{q}_{2}$ & $\tilde{q}_{3}$ & $\tilde{Q}_{1}$ & $\tilde{Q}_{2}$ & $\tilde{Q}_{3}$ & $I$ \\
\hline$\tilde{q}_{1}$ & -1 & $-\tilde{q}_{3}$ & $\tilde{q}_{2}$ & $-I$ & $-\tilde{Q}_{3}$ & $\tilde{Q}_{2}$ & $\tilde{Q}_{1}$ \\
\hline$\tilde{q}_{2}$ & $\tilde{q}_{3}$ & -1 & $-\tilde{q}_{1}$ & $\tilde{Q}_{3}$ & $-I$ & $-\tilde{Q}_{1}$ & $\tilde{Q}_{2}$ \\
\hline$\tilde{q}_{3}$ & $-\tilde{q}_{2}$ & $\tilde{q}_{1}$ & -1 & $-\tilde{Q}_{2}$ & $\tilde{Q}_{1}$ & $-I$ & $\tilde{Q}_{3}$ \\
\hline$\tilde{Q}_{1}$ & $I$ & $-\tilde{Q}_{3}$ & $\tilde{Q}_{2}$ & 1 & $\tilde{q}_{3}$ & $-\tilde{q}_{2}$ & $\tilde{q}_{1}$ \\
\hline$\tilde{Q}_{2}$ & $\tilde{Q}_{3}$ & $I$ & $-\tilde{Q}_{1}$ & $-\tilde{q}_{3}$ & 1 & $\tilde{q}_{1}$ & $\tilde{q}_{2}$ \\
\hline$\tilde{Q}_{3}$ & $-\tilde{Q}_{2}$ & $\tilde{Q}_{1}$ & $I$ & $\tilde{q}_{2}$ & $-\tilde{q}_{1}$ & 1 & $\tilde{q}_{3}$ \\
\hline$I$ & $-\tilde{Q}_{1}$ & $-\tilde{Q}_{2}$ & $-\tilde{Q}_{3}$ & $-\tilde{q}_{1}$ & $-\tilde{q}_{2}$ & $-\tilde{q}_{3}$ & 1 \\
\hline
\end{tabular}

Table 1: Octonion multiplication table

is anticommutative

$$
a b=-b a, \quad a, b=\tilde{q}_{i}, \tilde{Q}_{i}, I, \quad i=1,2,3
$$

and sometimes non-associative, for example

$$
\begin{aligned}
& \tilde{Q}_{m}\left(\tilde{Q}_{n} \tilde{Q}_{p}\right)=-\left(\tilde{Q}_{m} \tilde{Q}_{n}\right) \tilde{Q}_{p}, \quad m \neq n, n \neq p, p \neq m, \\
& \tilde{q}_{m}\left(\tilde{Q}_{n} \tilde{q}_{n}\right)=-\left(\tilde{q}_{m} \tilde{Q}_{n}\right) \tilde{q}_{n}, \\
& \left.\begin{array}{l}
\tilde{q}_{m}\left(\tilde{Q}_{m} \tilde{q}_{n}\right)=-\left(\tilde{q}_{m} \tilde{Q}_{m}\right) \tilde{q}_{n}, \quad \tilde{q}, \tilde{Q}_{m}\left(\tilde{q}_{n} \tilde{Q}_{n}\right)=-\left(\tilde{Q}_{m} \tilde{q}_{n}\right) \tilde{Q}_{n},
\end{array}\right\} \\
& \tilde{Q}_{m}\left(\tilde{q}_{n} \tilde{Q}_{n}\right)=-\left(\tilde{Q}_{m} \tilde{q}_{n}\right) \tilde{Q}_{n} \\
& \left.\tilde{Q}_{m}\left(\tilde{q}_{m} \tilde{Q}_{n}\right)=-\left(\tilde{Q}_{m} \tilde{q}_{m}\right) \tilde{Q}_{n}\right)
\end{aligned}
$$

The octonions designations in this paper $\tilde{q}_{i}, \tilde{Q}_{i}, i=1,2,3$ are coordinated with the octonions designations $e_{i}, i=1, \ldots, 7$ in Ref. [1] as follows:

$$
\tilde{q}_{1}=e_{1}, \tilde{q}_{2}=e_{3}, \tilde{q}_{3}=e_{7}, \tilde{Q}_{1}=i e_{5}, \tilde{Q}_{2}=i e_{4}, \tilde{Q}_{3}=i e_{2} \quad \text { here } i=\sqrt{-1}
$$

and with the designations in Ref. [4] as follows:

$$
\tilde{q}_{i}=j_{i}, \tilde{Q}_{i}=J_{i}, i=1,2,3 .
$$

\subsection{Non-associativity in Physics}

Probably the first attempt to apply the octonions in physics was the paper [2] describing an octonionic quantum mechanics. In Ref. [3] it is shown that some relation between octonions and string theory exists. An octonionic geometry in Ref. 4 is described. In this paper a new geometrical interpretation of the products of octonionic basis units is presented and eight real parameters of octonions are interpreted as the spacetime coordinates, momentum and energy. In Ref. [5] the non-associativity for the non-perturbative quantization of strongly interacting fields is introduced. A generalization of the quantum Hall effect where particles move in an eight dimensional space under an $S O(8)$ gauge field in Ref. [6] is considered. The underlying mathematics of this particle liquid is that of the last normed division algebra, the octonions. In Ref. [7] in the framework of non-associative geometry a unified description of continuum and discrete spacetime is proposed. In Ref(s). $\underline{8}$, 
[9] it is shown that for the generators of translations the Jacobi identity in the presence of the monopole fails and for the finite translations the operators $U(\vec{a}) \equiv \exp (i \vec{a} \hat{\vec{p}})$ do not associate. In Ref. 10 it is shown that non-associative extension of the group U(1) allows to obtain a consistent theory of point-like magnetic monopole with an arbitrary magnetic charge. In Ref. 11] a possible connection between quark confinement and octonions are investigated.

\section{A NON-ASSOCIATIVE QUANTUM MECHANICS}

In this section we would like to show that quantum mechanics can be based not only on the non-commutativity of operators but on the non-associativity, too. The main aim for such construction is that it can be a first step to building a non-perturbative quantum theory where the algebra of field operators is more complicated the algebra formed with the canonical non-commutative relationships.

Our basic idea is that the classical definition of an associator of three numbers $a, b, c$

$$
(a b) c-a(b c)=A s s[a(b c)]
$$

which measures the failure of associativity, can be generalized for operators

$$
(\hat{a} \hat{b}) \hat{c}-\hat{a}(\hat{b} \hat{c})=A s s[\hat{a}(\hat{b} \hat{c})] .
$$

where the quantity Ass $[\hat{a}(\hat{b} \hat{c})]$ is received from $A s s[a(b c)]$ at the replacement $a, b, c \rightarrow \hat{a}, \hat{b}, \hat{c}$ and quantum corrections are introduced. Of course the associator Ass $[\hat{a}(\hat{b} \hat{c})]$ depends on the product $\hat{a}(\hat{b} \hat{c})$.

For example, the standard quantization procedure (on the level of creation/annihilaion operators) can be presented as the destruction of the multiplication rule of real

$$
a b=b a
$$

or Grassman numbers

$$
a b=-b a
$$

by following wayt:

$$
\hat{a} \hat{b} \mp \hat{b} \hat{a}=1 .
$$

Following by this prescription we have to destroy the multiplication rules (B) for the product of two octonions and (4) (5) for the product of three octonions. For brevity we will omit the sign $\left(^{\wedge}\right)$. For the product of two operators with $i \neq j$ we will have

$$
\begin{aligned}
q_{i} q_{j} & =-q_{j} q_{i}, \\
q_{i} Q_{j} & =-Q_{j} q_{i}, \\
Q_{i} Q_{j} & =-Q_{j} Q_{i}
\end{aligned}
$$

and for the identical indexes

$$
q_{i} Q_{i}+Q_{i} q_{i}=1
$$

which is the ordinary canonical anti-commutation relationships for the creation/annihilation fermion operators and other anti-commutators are zero similar to ordinary quantum field theory. The associators/antiassociators for the product of three operators with different indexes $m \neq n, n \neq p, p \neq m$ are

$$
\begin{aligned}
Q_{m}\left(Q_{n} Q_{p}\right) & =-\left(Q_{m} Q_{n}\right) Q_{p}+\epsilon_{m n p} \mathcal{H}_{3} \\
q_{m}\left(q_{n} q_{p}\right) & =\left(q_{m} q_{n}\right) q_{p}+\epsilon_{m n p} \mathcal{H}_{3} \\
q_{m}\left(Q_{n} q_{p}\right) & =-\left(q_{m} Q_{n}\right) q_{p}+\epsilon_{m n p} \mathcal{H}_{3} \\
q_{m}\left(q_{n} Q_{p}\right) & =\left(q_{m} q_{n}\right) Q_{p}+\epsilon_{m n p} \mathcal{H}_{3} \\
Q_{m}\left(q_{n} q_{p}\right) & =\left(Q_{m} q_{n}\right) q_{p}+\epsilon_{m n p} \mathcal{H}_{3} \\
q_{m}\left(Q_{n} Q_{p}\right) & =\left(q_{m} Q_{n}\right) Q_{p}+\epsilon_{m n p} \mathcal{H}_{3} \\
Q_{m}\left(q_{n} Q_{p}\right) & =\left(Q_{m} q_{n}\right) Q_{p}+\epsilon_{m n p} \mathcal{H}_{3} \\
Q_{m}\left(Q_{n} q_{p}\right) & =\left(Q_{m} Q_{n}\right) q_{p}+\epsilon_{m n p} \mathcal{H}_{3}
\end{aligned}
$$


where $\mathcal{H}_{3}$ is for the time undefined operator, the index 3 of the operator $\mathcal{H}_{3}$ means that we consider the product of three operators. The associators/anti-associators for the product of three operators as $q(Q q)$ and $Q(q Q)$ and with two different indexes $m \neq n$ we have

$$
\begin{aligned}
q_{m}\left(Q_{n} q_{n}\right) & =-\left(q_{m} Q_{n}\right) q_{n}+q_{m}, \\
q_{m}\left(Q_{m} q_{n}\right) & =-\left(q_{m} Q_{m}\right) q_{n}+q_{n}, \\
Q_{m}\left(q_{n} Q_{n}\right) & =-\left(Q_{m} q_{n}\right) Q_{n}+Q_{m}, \\
Q_{m}\left(q_{m} Q_{n}\right) & =-\left(Q_{m} q_{m}\right) Q_{n}+Q_{n} .
\end{aligned}
$$

For the product of three operators as $q(Q Q)$ and $Q(q q)$ and with two different indexes $m \neq n$ we have

$$
\begin{aligned}
q_{m}\left(Q_{m} Q_{n}\right) & =\left(q_{m} Q_{m}\right) Q_{n}+\mathcal{H}_{3,1} \\
q_{m}\left(Q_{n} Q_{m}\right) & =\left(q_{m} Q_{n}\right) Q_{m}+\mathcal{H}_{3,1}^{\prime} \\
Q_{m}\left(q_{m} q_{n}\right) & =\left(Q_{m} q_{m}\right) q_{n}+\mathcal{H}_{3,2} \\
Q_{m}\left(q_{n} q_{m}\right) & =\left(Q_{m} q_{n}\right) q_{m}+\mathcal{H}_{3,2}^{\prime} \\
Q_{m}\left(Q_{n} q_{m}\right) & =\left(Q_{m} Q_{n}\right) q_{m}+\mathcal{H}_{3,3} \\
Q_{n}\left(Q_{m} q_{m}\right) & =\left(Q_{n} Q_{m}\right) q_{m}+\mathcal{H}_{3,3}^{\prime} \\
q_{m}\left(q_{n} Q_{m}\right) & =\left(q_{m} q_{n}\right) Q_{m}+\mathcal{H}_{3,4} \\
q_{n}\left(q_{m} Q_{m}\right) & =\left(q_{n} q_{m}\right) Q_{m}+\mathcal{H}_{3,4}^{\prime}
\end{aligned}
$$

The second index $i$ of $\mathcal{H}_{3, i}$ and $\mathcal{H}_{3, i}^{\prime}$ operators means that the corresponding associators (for example, $\left.q_{m}\left(Q_{m} Q_{n}\right)-\left(q_{m} Q_{m}\right) Q_{n}=\mathcal{H}_{3,1}\right)$ can be different. We will retain the alternativity property of the octonions

$$
\begin{aligned}
q_{n}^{2} Q_{m} & =\left(q_{n} q_{n}\right) Q_{m}=q_{n}\left(q_{n} Q_{m}\right) \\
q_{n}\left(Q_{m} q_{n}\right) & =\left(q_{n} Q_{m}\right) q_{n}, \\
Q_{m} q_{n}^{2} & =Q_{m}\left(q_{n} q_{n}\right)=\left(Q_{m} q_{n}\right) q_{n} \\
Q_{n}^{2} q_{m} & =\left(Q_{n} Q_{n}\right) q_{m}=Q_{n}\left(Q_{n} q_{m}\right) \\
Q_{n}\left(q_{m} Q_{n}\right) & =\left(Q_{n} q_{m}\right) Q_{n} \\
q_{m} Q_{n}^{2} & =q_{m}\left(Q_{n} Q_{n}\right)=\left(q_{m} Q_{n}\right) Q_{n}
\end{aligned}
$$

The associativity rule for the product of four and more operators will be

$$
\hat{a}(\hat{b} \hat{c})= \pm(\hat{a} \hat{b}) \hat{c}+\mathcal{H}_{n}[\hat{a}(\hat{b} \hat{c})]
$$

where the operators $\hat{a}, \hat{b}, \hat{c}$ can be any product of the operators $q_{i}, Q_{j} ; \mathcal{H}_{n}[\hat{a}(\hat{b} \hat{c})]$ is some operator and the sign $(+)$ or $(-)$ in front of the first term of the right hand side of Eq. (43) is defined from the multiplication of the corresponding octonions

$$
a(b c)= \pm(a b) c
$$

where $a, b, c$ are the octonions with the same product of the octonions $\tilde{q}_{i}, \tilde{Q}_{j}$ as in Eq. (43).

One of the main problem in such non-associative algebra with the definitions (13)-(42) is the proof of the self-consistency of such algebra. This is a very complicated problem and we will check the self-consistency for the products of three operators only.

\section{The Products of Three Operators}

At first we will check the non-associativity relationship (17). For this we will permute the first and third factors in the product

$$
\begin{aligned}
Q_{1}\left(Q_{2} Q_{3}\right) & =-Q_{1}\left(Q_{3} Q_{2}\right)=\left(Q_{1} Q_{3}\right) Q_{2}+\mathcal{H}_{3}=-\left(Q_{3} Q_{1}\right) Q_{2}+\mathcal{H}_{3} \\
& =Q_{3}\left(Q_{1} Q_{2}\right)=-Q_{3}\left(Q_{2} Q_{1}\right) .
\end{aligned}
$$

On the other hand

$$
\begin{aligned}
Q_{1}\left(Q_{2} Q_{3}\right) & =-\left(Q_{1} Q_{2}\right) Q_{3}+\mathcal{H}_{3}=\left(Q_{2} Q_{1}\right) Q_{3}+\mathcal{H}_{3}=-Q_{2}\left(Q_{1} Q_{3}\right) \\
& =Q_{2}\left(Q_{3} Q_{1}\right)=-\left(Q_{2} Q_{3}\right) Q_{1}+\mathcal{H}_{3}=\left(Q_{3} Q_{2}\right) Q_{1}+\mathcal{H}_{3} \\
& =-Q_{3}\left(Q_{2} Q_{1}\right) .
\end{aligned}
$$


The same is correct for any permutation of the indexes $(1,2,3)$. Analogous calculations can be made for the product with $q_{1}, q_{2}, q_{3}$

$$
q_{1}\left(q_{2} q_{3}\right)=-q_{3}\left(q_{2} q_{1}\right) .
$$

The calculations for three different indexes but with two $q$ and one $Q$

$$
q_{1}\left(Q_{2} q_{3}\right)=-q_{3}\left(Q_{2} q_{1}\right) .
$$

The same calculations for two $Q$ and one $q$

$$
Q_{1}\left(q_{2} Q_{3}\right)=-Q_{3}\left(q_{2} Q_{1}\right) .
$$

$\mathrm{Eq}(\mathrm{s})$. (46)-(49) are correct for any permutation of the indexes $(1,2,3)$. The analogous calculations with two equal indexes give us

$$
\begin{aligned}
q_{1}\left(Q_{1} Q_{2}\right)= & Q_{2}\left(Q_{1} q_{1}\right)-\mathcal{H}_{3,1}^{\prime}=Q_{2}\left(Q_{1} q_{1}\right)+\mathcal{H}_{3,1}-\mathcal{H}_{3,3}-\mathcal{H}_{3,3}^{\prime}, \\
Q_{1}\left(q_{1} Q_{2}\right)= & -Q_{2}\left(q_{1} Q_{1}\right)-\mathcal{H}_{3,1}-\mathcal{H}_{3,1}^{\prime}+i \hbar Q_{2}= \\
& -Q_{2}\left(q_{1} Q_{1}\right)-\mathcal{H}_{3,3}-\mathcal{H}_{3,3}^{\prime}+i \hbar Q_{2} \\
Q_{1}\left(q_{1} q_{2}\right)= & q_{2}\left(q_{1} Q_{1}\right)-\mathcal{H}_{3,2}^{\prime}=q_{2}\left(q_{1} Q_{1}\right)+\mathcal{H}_{3,2}-\mathcal{H}_{3,4}-\mathcal{H}_{3,4}^{\prime}, \\
q_{1}\left(Q_{1} q_{2}\right)= & -q_{2}\left(Q_{1} q_{1}\right)-\mathcal{H}_{3,4}-\mathcal{H}_{3,4}^{\prime}+i \hbar q_{2}= \\
& -q_{2}\left(Q_{1} q_{1}\right)-\mathcal{H}_{3,2}-\mathcal{H}_{3,2}^{\prime}+i \hbar q_{2} .
\end{aligned}
$$

Consequently on the level of the 3-product one can say only that

$$
\begin{aligned}
\mathcal{H}_{3,1}+\mathcal{H}_{3,1}^{\prime} & =\mathcal{H}_{3,3}+\mathcal{H}_{3,3}^{\prime}, \\
\mathcal{H}_{3,2}+\mathcal{H}_{3,2}^{\prime} & =\mathcal{H}_{3,4}+\mathcal{H}_{3,4}^{\prime} .
\end{aligned}
$$

The pair of indexes $(1,2)$ in Eq(s). (501)- (553) can be replaced by any other pair indexes $(i, j)$. The selfconsistency of the relations (37)-(39) follows from the consistency of the multiplication of the octonions.

On the level of the product of three operators we can not define the operators $\mathcal{H}_{3, i}, i=1,2,3,4$. But comparing with the relation (16) one can suppose that

$$
\mathcal{H}_{3, i}=\hbar_{2} \sum_{j}\left(\alpha_{i, j} q_{j}+\beta_{i, j} Q_{j}\right)
$$

where $\hbar_{2}$ is a constant which can be called the second Planck constant and $\alpha_{i, j}, \beta_{i, j}$ are some constants.

\section{SOME PROPERTIES OF THE NON-ASSOCIATIVE QUANTUM MECHANICS}

For the octonions $q_{i}, Q_{i}$ we have the following relation between $\tilde{q}_{i}$ and $\tilde{Q}_{i}$

$$
\tilde{q}_{i} \tilde{Q}_{i}-\tilde{Q}_{i} \tilde{q}_{i}=2 I .
$$

This allows us to suppose that the corresponding operators are similar to canonical conjugate variables and have to have the anti-commutation relationships (16).

One can see that there is some similarity with between $q_{i}, Q_{j}$ and the annihilation and creation operators of quarks. Every value of index $i=1,2,3$ corresponds to some color $=$ red,green,blue. In this case the operators $q_{i}, Q_{i}$ correspond to the annihilation and creation operators of quarks. But this similarity does not mean the equivalence as the non-associative quantum mechanics should describe non-free (strongly interacting) particles.

In the standard quantum mechanics for the harmonic oscillator one can introduce the annihilation operator $\hat{a}$ such that

$$
\hat{a}\left|v a c_{o s c}\right\rangle=0
$$

where $\left|v a c_{o s c}\right\rangle$ is a vacuum state for the oscillator. But for any another potential

$$
\hat{a}\left|v a c_{\text {nonosc }}\right\rangle \neq 0
$$


Consequently for the non-associative quantum mechanics we also have

$$
q_{i}\left|v a c_{\text {nonass }}\right\rangle \neq 0 .
$$

Probably it is similar to the existence of a condensate in quantum chromodynamics. Following the abovementioned correspondence one can introduce "colorless" operators $q_{1}\left(q_{2} q_{3}\right), Q_{1}\left(Q_{2} Q_{3}\right),\left(q_{i} Q_{i}\right)$. These operators can be named by the following manner

$$
\begin{gathered}
q_{1}\left(q_{2} q_{3}\right) \text { - "nucleon" annihilation operator, } \\
Q_{1}\left(Q_{2} Q_{3}\right) \text { - "nucleon" creation operator, } \\
Q_{i} q_{i}-\text { "meson" annihilation operator, } \\
q_{i} Q_{i} \text { - "meson" creation operator, } \\
q_{i} q_{i} \text { - "Cooper pair" annihilation operator, } \\
Q_{i} Q_{i} \text { - "Cooper pair" creation operator. }
\end{gathered}
$$

\subsection{The Commutation Relationship for "meson"}

Let us consider the commutator

$$
\left(q_{i} q_{i}\right)\left(Q_{i} Q_{i}\right)-\left(Q_{i} Q_{i}\right)\left(q_{i} q_{i}\right)=?
$$

on $i$ there is no summation. As before we will suppose that the products of four operators are alternative

$$
\begin{aligned}
q_{1}\left(q_{1} Q_{1}^{2}\right) & =q_{1}^{2} Q_{1}^{2}=\left(q_{1}^{2} Q_{1}\right) Q_{1}, \\
Q_{1}\left(Q_{1} q_{1}^{2}\right) & =Q_{1}^{2} q_{1}^{2}=\left(Q_{1}^{2} q_{1}\right) q_{1}, \\
q_{1}\left(Q_{1}^{2} q_{1}\right) & =\left(q_{1} Q_{1}^{2}\right) q_{1} .
\end{aligned}
$$

For the another order of the factors

$$
\begin{aligned}
& q_{1}\left(Q_{1}\left(q_{1} Q_{1}\right)\right)=\left(q_{1} Q_{1}\right)\left(q_{1} Q_{1}\right)+\mathcal{H}_{4,1}, \\
& \left(\left(q_{1} Q_{1}\right) q_{1}\right) Q_{1}=\left(q_{1} Q_{1}\right)\left(q_{1} Q_{1}\right)+\mathcal{H}_{4,1}^{\prime}, \\
& Q_{1}\left(q_{1}\left(Q_{1} q_{1}\right)\right)=\left(Q_{1} q_{1}\right)\left(Q_{1} q_{1}\right)+\mathcal{H}_{4,2}, \\
& \left(\left(Q_{1} q_{1}\right) Q_{1}\right) q_{1}=\left(Q_{1} q_{1}\right)\left(Q_{1} q_{1}\right)+\mathcal{H}_{4,2}^{\prime} .
\end{aligned}
$$

Following to the different ways of the brackets arrangement we have

$$
\begin{aligned}
q_{1}^{2} Q_{1}^{2} & =Q_{1}^{2} q_{1}^{2}-\mathcal{H}_{4,1}+\mathcal{H}_{4,2}=Q_{1}^{2} q_{1}^{2}-\mathcal{H}_{4,1}+\mathcal{H}_{4,2}^{\prime} \\
& =Q_{1}^{2} q_{1}^{2}-\mathcal{H}_{4,1}^{\prime}+\mathcal{H}_{4,2}^{\prime}=Q_{1}^{2} q_{1}^{2}-\mathcal{H}_{4,1}^{\prime}+\mathcal{H}_{4,2},
\end{aligned}
$$

i.e.

$$
\mathcal{H}_{4,1}=\mathcal{H}_{4,1}^{\prime}, \quad \mathcal{H}_{4,2}=\mathcal{H}_{4,2}^{\prime}
$$

and

$$
q_{1}^{2} Q_{1}^{2}-Q_{1}^{2} q_{1}^{2}=-\mathcal{H}_{4,1}+\mathcal{H}_{4,2} .
$$

One can suppose that the difference $\mathcal{H}_{4,1}-\mathcal{H}_{4,2}$ can be defined demanding the self-consistency of the product of five operators and so on.

\section{CONCLUSIONS AND DISCUSSION}

In this notice it is shown that one can try to destroy the octonions multiplication rules to receive a nonassociative quantum mechanics which is not equivalent to the standard quantum mechanics. The distinction is that the algebra of operators is generated not only by the canonical commutation/anti-commutations relationships but also by the rules regulating the brackets order in the operators product. The construction of such quantum mechanics is very complicated problem and now we would like to list some unresolved problems :

1. In this notice we show the self-consistency of the product of three operators. But the self-consistency of the product of four and more operators is an open problem. 
2. Non-associative operators can not have the representation on the functions like $\hat{p}_{x}=-i \hbar \frac{\partial}{\partial x}$ in the standard quantum mechanics. In this context the question is how one can define eigenstates and eigenfunctions and what are observables of the non-associative operators?

3. Probably such non-associative quantum mechanics is hardly realized in the Nature but one can presuppose that some generalization of the non-associative quantum mechanics for the quantum field theory can exist. The matter is that only for free fields the algebra of quantized fields is known. The algebra of strongly interacting quantum fields (in some regimes of quantum chromodynamics, quantum gravity and so on) remains unknown. It means that the algebra of operators of the strongly interacting fields is much more complicated the algebra of operators generating only by the canonical commutation/anti-commutation relationships and probably the algebra of strongly interacting fields can be non-associative one.

4. One can presuppose that the formulation of the rules for the rearrangement of brackets in some nonassociative operators product will lead to the appearance of a second Planck constant.

\section{REFERENCES}

[1] J. C. Baez, "The Octonions," Bull. Amer. Math. Soc., 39, 145-205 (2002), math.ra/0105155

[2] Pascual Jordan, John von Neumann, Eugene Wigner, "On an algebraic generalization of the quantum mechanical formalism," Ann. Math. 35, 29-64 (1934).

[3] T. Kugo and P.-K. Townsend, "Supersymmetry and the division algebras," Nucl. Phys. B221, 357-380 (1983).

[4] M. Gogberashvili, "Octonionic Geometry," hep-th/0409173

[5] V. Dzhunushaliev, "Non-perturbative operator quantization of strongly interacting fields," Found. Phys. Lett. 16, 57 (2003).

[6] B. A. Bernevig, J. p. Hu, N. Toumbas and S. C. Zhang, "The Eight Dimensional Quantum Hall Effect and the Octonions," Phys. Rev. Lett. 91, 236803 (2003).

[7] A. I. Nesterov and L. V. Sabinin, "Nonassociative geometry: Towards discrete structure of spacetime," Phys. Rev. D62, 081501 (2000).

[8] B. Grossman, "A Three Cocycle in Quantum Mechanics," Phys. Lett. B152, 93, (1985).

[9] R. Jackiw, "3 - Cocycle in Mathematics and Physics," Phys. Rev. Lett. 54, 159, (1985).

[10] A. I. Nesterov, "Three-cocycles, nonassociative gauge transformations and Dirac's monopole," Phys. Lett. A328, 110 (2004).

[11] M. Günaydin and F. Gürsey "Quark Structure and Octonions," J. Math. Phys. 14, 1651 (1973); “ Quark Statistics and Octonions," Phys. Rev. D9, 3387 (1974). 